\title{
Role of Drosophila EDEMs in the degradation of the alpha-1-antitrypsin $Z$ variant
}

\author{
BO-YUN JANG ${ }^{1}$, HYUNG DON RYOO ${ }^{3}$, JAEKYOUNG SON ${ }^{1,2}$, KYUNG-CHUL CHOI ${ }^{1,2}$, \\ DONG-MYOUNG SHIN ${ }^{1}$, SANG-WOOK KANG ${ }^{1,2}$ and MIN-JI KANG ${ }^{1,2}$
}

\author{
${ }^{1}$ Department of Biomedical Sciences, and ${ }^{2}$ Cell Dysfunction Research Center and Bio-Medical Institute of Technology (BMIT), \\ University of Ulsan College of Medicine, Seoul 138-736, Republic of Korea; ${ }^{3}$ Department of Cell Biology, \\ New York University School of Medicine, New York, NY 10016, USA
}

Received December 2, 2014; Accepted February 18, 2015

DOI: $10.3892 /$ ijmm.2015.2109

\begin{abstract}
The synthesis of proteins in the endoplasmic reticulum (ER) that exceeds the protein folding capacity of this organelle is a frequent cause of cellular dysfunction and disease. An example of such a disease is alpha-1-antitrypsin (A1AT) deficiency, caused by destabilizing mutations in this glycoprotein. It is considered that the mutant proteins are recognized in the ER by lectins and are subsequently degraded through the proteasome, leading to a deficiency in this enzyme in the afflicted patients. We previously established a Drosophila model of this disease by overexpressing the null Hong Kong (NHK) allele of this gene and found that the Drosophila lectin, ER degradation-enhancing $\alpha$-mannosidase-like protein 2 (EDEM2), can accelerate the degradation of A1AT when overexpressed. NHK is a rare allele, and in this study, we investigated in depth the mechanisms through which Drosophila EDEMs affect the degradation of the $Z$ variant, which is the predominant disease allele. Specifically, we report that the $\mathrm{Z}$ allele does not activate ER stress signaling as prominently as the NHK allele, but similarly requires both Drosophila EDEM1 and EDEM2 for the degradation of the protein. We demonstrate that EDEMs are required for their ubiquitination, and without EDEMs, glycosylated A1AT mutants accumulate in cells. These results support the role of the EDEM-mediated ubiquitination of the alpha-1-antitrypsin Z (ATZ) allele, and establish a Drosophila model for the study of this protein and disease.
\end{abstract}

\section{Introduction}

The endoplasmic reticulum(ER) is the cellular organellein which membrane and secretory proteins are synthesized, glycosylated

Correspondence to: Professor Min-Ji Kang, Department of Biomedical Sciences, University of Ulsan College of Medicine, 88 Olympic-ro 43-gil, Songpa-gu, Seoul 138-736, Republic of Korea E-mail: mjkang@amc.seoul.kr

Key words: endoplasmic reticulum stress, unfolded protein response, endoplasmic reticulum degradation-enhancing $\alpha$-mannosidase-like protein, alpha-1-antitrypsin deficiency, Z variant of alpha-1-antitrypsin, null Hong Kong and acquire their correct conformation. While the properly folded proteins can leave the ER and traffick to their final destination along the secretary pathway, proteins that fail to fold are retrotranslocated to the cytoplasm for degradation, a process that is referred to as ER-associated degradation (ERAD) (1). ERAD is initiated by substrate recognition in the ER, followed by retrotranslocation into the cytoplasm for ubiquitin-mediated proteasomal degradation. For ERAD to occur properly, a machinery that can recognize misfolded proteins is required. While ER degradation-enhancing $\alpha$-mannosidase-like proteins (EDEMs) were initially considered lectins $(2,3)$, recent studies have revealed that EDEMs can function as mannosidases $(4,5)$ and molecular chaperones (6). We have previously reported that the Drosophila genome encodes 2 EDEM homologs, EDEM1 (CG3810) and EDEM2 (CG5682) (14). Sequence analysis indicated that whereas Drosophila EDEM1 was similar to human EDEM2, Drosophila EDEM2 showed a closer resemblance to EDEM3 in mammals (Fig. 1).

Proteins that misfold in the ER underlie a number of conformational diseases in humans. Among these diseases is alpha-1-antitrypsin (A1AT) deficiency, which is caused by mutations in the A1AT gene that impairs its protein folding properties during biogenesis. The classical form of mutant A1AT protein is alpha-1-antitrypsin Z (ATZ) that results from a Glu342Lys substitution, rendering it prone to polymerization and aggregation (7). Misfolded ATZ is rapidly cleared from cells, through a combination of ERAD (8-10) and autophagy (11-13).

Previously, we established a Drosophila model to study how A1AT is degraded through ERAD (14). In that previous study, we had focused on the rare null Hong Kong (NHK) allele $(2,3,15)$, and we had shown that the overexpression of Drosophila EDEM2 promotes ERAD of NHK. In this study, we performed a more in-depth investigation of Drosophila EDEMs, focusing on the predominant disease allele Z. Specifically, we demonstrate that the two Drosophila EDEMs play redundant roles in the degradation of the $\mathrm{Z}$ allele. We also demonstrate that the knockdown of these two genes leads to the accumulation of glycosylated ATZ proteins, while the overexpression of EDEMs promotes the degradation of ATZ. In addition, we provide evidence of A1AT ubiquitination, using cell-based ubiquitination assays. 

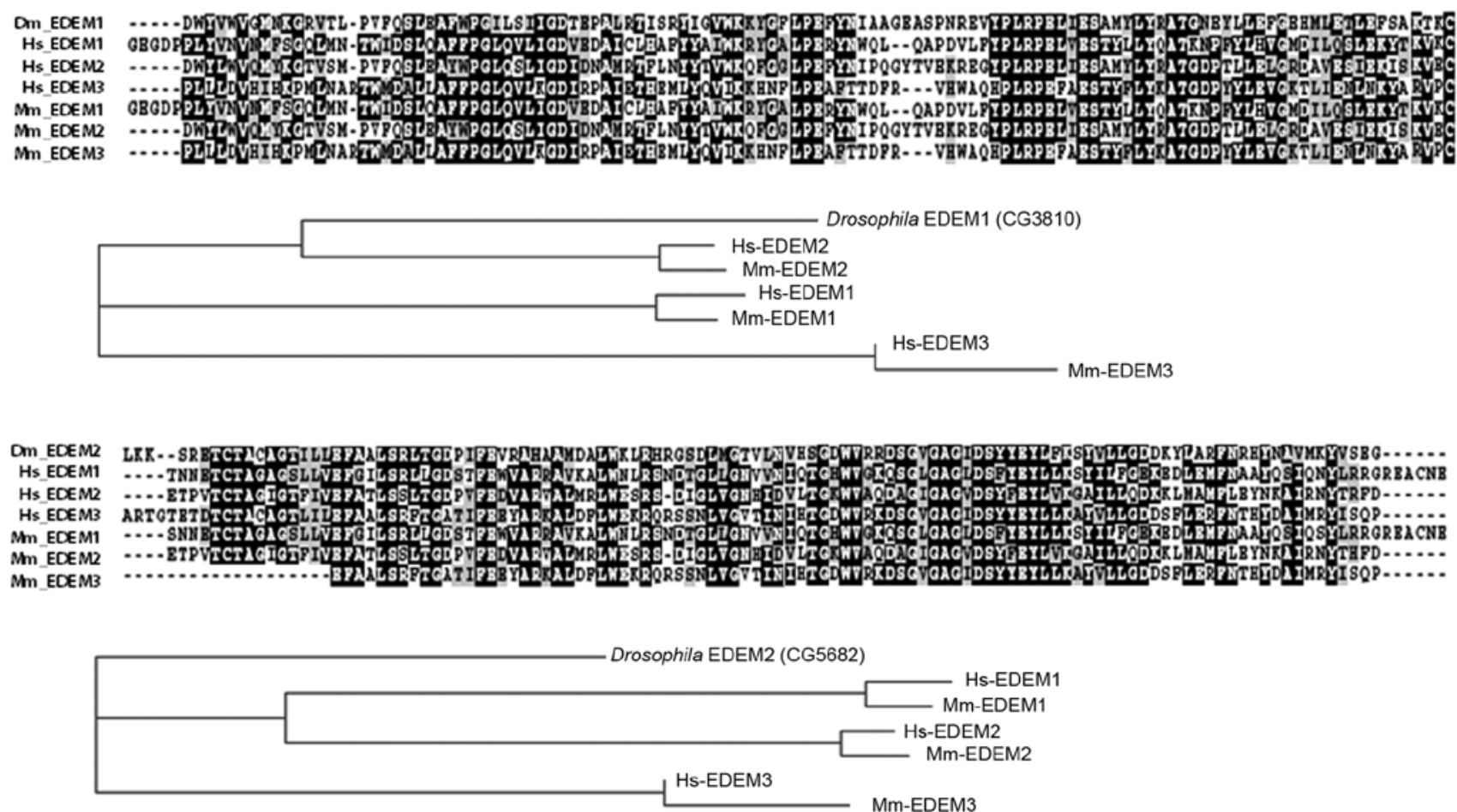

Figure 1. Alignment of the endoplasmic reticulum degradation-enhancing $\alpha$-mannosidase-like protein (EDEM)1 and EDEM2 genes from Drosophila with the homologous proteins from human and mouse EDEM1-3. The intensity of darkness denotes the extent of conservation.

\section{Materials and methods}

Plasmids and fly stocks. Genes were expressed in Drosophila through the standard GAL4/UAS system (16). The following flies and DNA have been described previously: armadillo-GAL4 (17), uas-myc-EDEM1 (14), uas-myc-EDEM2 (14) and uas-NHK (14). The DNA encoding ATZ (18) was subcloned into a pUAST plasmid.

Cell culture and RNAi treatment. Drosophila Schneider 2 (S2) cells were grown in Schneider's medium supplemented with $10 \%$ fetal bovine serum and $1 \%$ penicillin/streptomycin (Invitrogen, Grand Island, NY, USA). Treatment with doublestranded RNA (dsRNA) was performed as described in a previous study (19). Briefly, the cells were plated into 6-well plates at a density of $1 \times 10^{6}$ cells/well before treatment with dsRNA (day 1). After $24 \mathrm{~h}, 20 \mu \mathrm{g}$ of EGFP, EDEM1 or EDEM2 dsRNA were added to each well following another boost with $20 \mu \mathrm{g}$ dsRNA on day 4 . The cells were then transiently transfected with either NHK or ATZ using Effectene ${ }^{\mathrm{TM}}$ (Qiagen, Valencia, CA, USA) on day 5. The cells were split on day 8 and lysed to examine the level of NHK or ATZ on day 9 . The EDEM1 dsRNA consisted of a 516-nt region (Amplicon ID: DRSC18573), as described by the Drosophila RNAi Screening Center (http://www.flyrnai.org). The following primers were used to amplify this sequence from an embryo cDNA library: 'R' primer, CAATGTTGTCACCCACGAAA; 'S' primer, TCGAAGTT GCTTACTAACAGA. This amplicon has no predicted off-targets. The EDEM2 dsRNA consisted of a 520-nucleotide region (amplicon ID: DRSC02877). The following primers were used to amplify this sequence from an embryo cDNA library: 'R' primer, 5'-CATGCGCGGGTTAAT CTC-3'; 'S' primer, 5'-GATAGAGCATCTCGTGTGTC-3'. To induce ER stress, Drosophila S2 cells were treated with dithiothreitol (DTT; Cat. no. 43815; Sigma, St. Louis, MO, USA) or thapsigargin (Tg; Cat. no. T9033; Sigma) for the indicated periods of time.

Immunohistochemistry. All fluorescent images were captured under a Zeiss LSM 510 confocal microscope, using x100 objective lenses. The following antibodies were used: guinea pig anti-Hsc3 antibody (1:500) as previously described (17), mouse anti-myc (1:1,000 for immunohistochemistry; 9E10; Cat. no. 11667149001; Roche Diagnostics GmbH, Mannheim, Germany), rhodamine red anti-mouse secondary antibody (Cat. no. 715-295-150; 1:500) and FITC anti-guinea pig secondary antibody (Cat. no. 706-095-148; 1:500) (both from Jackson ImmunoResearch, West Grove, PA, USA).

Western blot analysis and immunoprecipitation. For western blot analyses, Drosophila S2 cells were extracted with 1\% SDS lysis buffer (10 mM Tris pH 7.5, $1 \mathrm{mM}$ EDTA, $150 \mathrm{mM} \mathrm{NaCl}$ and $1 \%$ SDS; Sigma). Following centrifugation at $16,100 \mathrm{x} g$ for $10 \mathrm{~min}$, the supernatants were fractionated by SDS-PAGE and transferred onto polyvinylidene difluoride (PVDF) membranes (Millipore, Billerica, MA, USA). The membranes were then probed with the indicated antibodies: polyclonal rabbit antiA1AT (1:5,000 for western blot analysis; Cat. no. A0012; Dako, Glostrup, Denmark), mouse anti-profilin (1:2,000 for western blot analysis; Developmental Studies Hybridoma Bank, chi 1J, University of Iowa, Iowa City, IA, USA), rat anti-HA antibody (Cat. no. 11867423001; 1:1,000; Roche Diagnostics GmbH) 


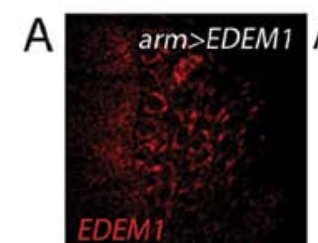

$\mathrm{B}$

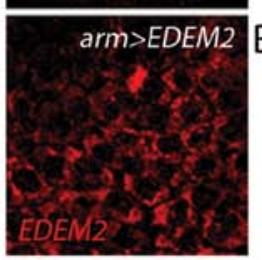

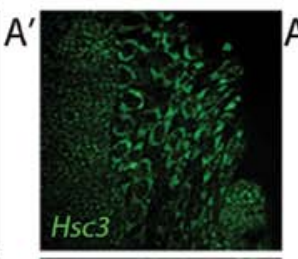
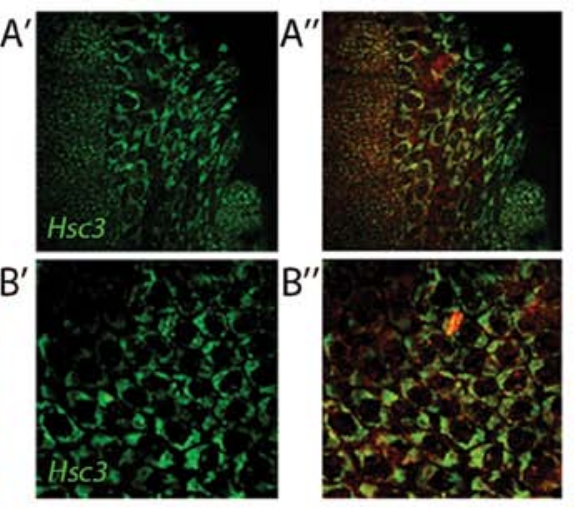

C $1 \mathrm{mMDTT}$ NT $\frac{8 \mathrm{~h}}{-+} \frac{12 \mathrm{~h}}{-+}$

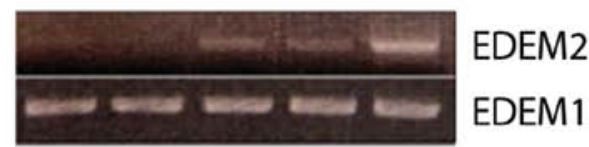

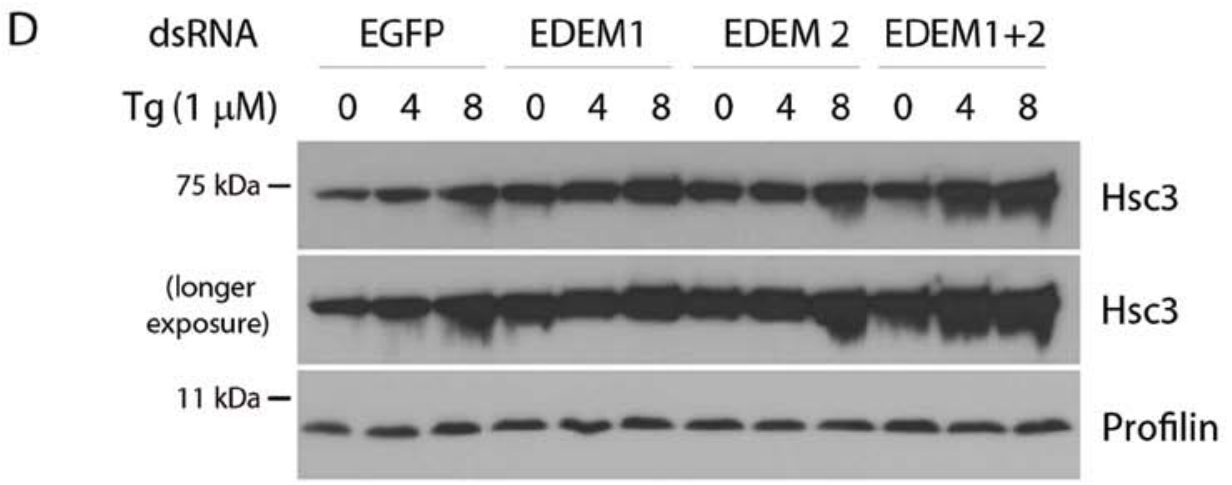

Figure 2. Characterization of Drosophila endoplasmic reticulum (ER) degradation-enhancing $\alpha$-mannosidase-like proteins (EDEMs). (A and B) Subcellular localization of EDEMs. Immunohistochemistry of the embryonic amnioserosa cells revealed that myc-tagged EDEM protein (red) co-localizes with the ER resident chaperone Hsc3 (green). (C) Induction of EDEM2 expression by ER stress. Drosophila cells were treated with 1 mM dithiothreitol (DTT) for the indicated periods of time. The transcription of EDEM2, but not that of EDEM1, was induced by ER stress. NT, not treated (D) Hsc3 induction by thapsigargin (Tg) treatment increased after the knockdown of EDEM1 or EDEM2. The upper and middle panels show anti-Hsc3 western blots, whereas the lower panel shows anti-profilin as a loading control.

and rabbit anti-GFP antibody (1:5,000; Cat. no. A6455; Molecular Probes, Eugene, OR, USA). The fractionation of the Drosophila S2 cells was performed as previously described (20). For immunoprecipitation, the Drosophila $\mathrm{S} 2$ cells were extracted with $1 \%$ Triton X-100 lysis buffer (50 mM Tris pH 8.0, $150 \mathrm{mM}$ $\mathrm{NaCl}$, digitonin and $1 \%$ Triton X-100; Sigma) for $20 \mathrm{~min}$ on ice, and centrifuged at $16,100 \mathrm{x}$. The supernatant was quantified by Bradford assay, and used for immunoprecipitation. Immunoprecipitation was performed with anti-A1AT antibody and protein-G-agarose beads (Roche Diagnostics $\mathrm{GmbH}$ ). The beads were washed 3 times in low-ionic-strength buffer $(50 \mathrm{mM}$ Tris-Cl pH 8.0, $100 \mathrm{mM} \mathrm{NaCl}$ and 1\% Triton X-100; Sigma). Rat anti-HA antibody was used to detect the ubiquitination of A1AT. For quantification of western bands, we used ImageJ software (http://rsbweb.nih.gov/ij). The intensity of the band of interest was normalized with an anti-profilin band.

Measurement of RNA levels. Total RNA was isolated using TRIzol reagent, and reverse transcription was performed from $200 \mathrm{ng}$ of total RNA using the SuperScript First-Strand Synthesis kit (both from Invitrogen). The following primer sequences were used for amplification and quantification: dEDEM1-F, ACGCCTACGATGGTTACCTG; dEDEM1-R, ACACGTTGATGTCCCTGTCA; dEDEM2-F, CTTAGCA CCGAAACCACCAT; dEDEM2-R, ACTCCTCGGTACCG TCCTTT.

\section{Results}

Characterization of Drosophila EDEMs. To determine the subcellular distribution of EDEMs in Drosophila, we generated transgenic EDEM lines with epitope tags and expressed them in Drosophila embryo amnioserosa cells. Immunolabeling with anti-myc antibody revealed that the Drosophila EDEMs co-localized with Hsc3, the Drosophila orthologue of mammalian BIP. This result is consistent with the hypothesis that Drosophila EDEMs reside in the ER (Fig. 2A and B).

Mammalian EDEMs are stress-regulated proteins that are induced by ER stress $(21,22)$. To determine whether the Drosophila EDEM homologs are similarly regulated, we treated the Drosophila S2 cells with dithiothreitol (DTT), which imposes ER stress by reducing disulfide bonds. Under these conditions, Drosophila EDEM2 expression was transcriptionally induced (Fig. 2C). Similar results were obtained with independent ER stress-causing chemicals; tunicamycin $(10 \mu \mathrm{g} / \mathrm{ml})$, which inhibits the glycosylation of proteins in the ER and thapsigargin (Tg; $1 \mu \mathrm{M}$ ), which perturbs ER-calcium homeostasis (data not shown). On the other hand, we were not able to detect the induction of Drosophila EDEM1 under these conditions.

The degree of ER stress in cells can be indirectly assessed by the extent of the transcriptional response that induces ER chaperones and ERAD genes. To examine the protective role of 
A

\begin{tabular}{|c|c|c|c|c|c|}
\hline NHK & - & + & - & + & - \\
\hline ATZ & - & - & + & - & + \\
\hline XBP1-EGFP & + & - & - & + & + \\
\hline $1 \mathrm{mM}$ DTT & - & - & - & - & - \\
\hline
\end{tabular}

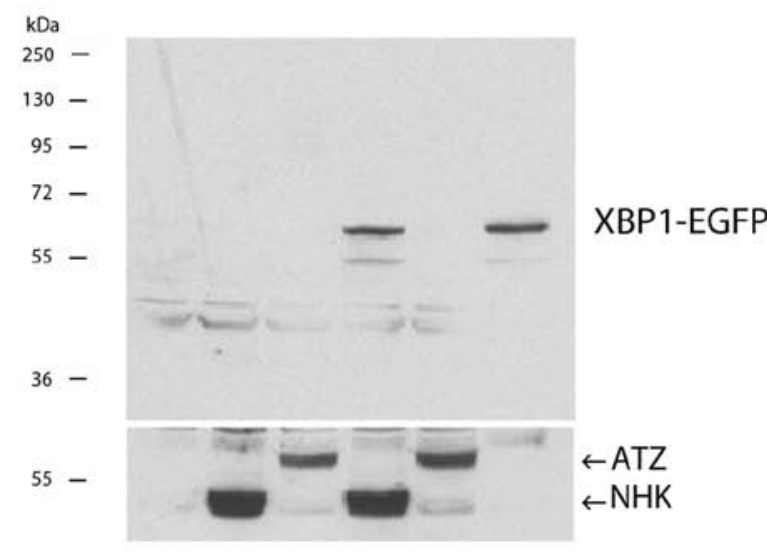

B

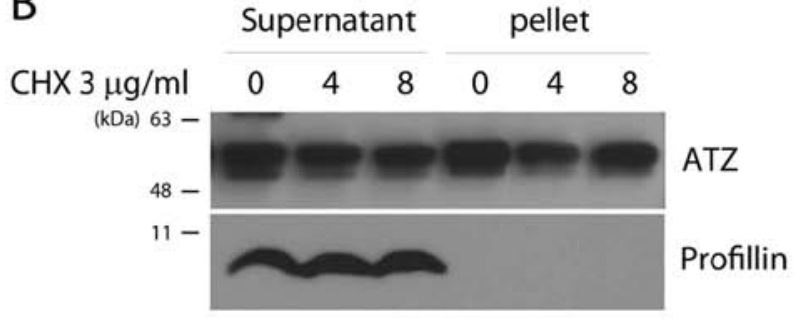

C

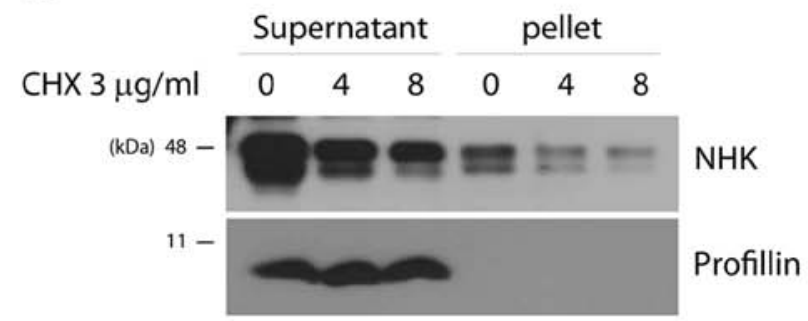

Figure 3. The Z variant of $\alpha$-1-antitrypsin Z (ATZ) does not activate X-box binding protein 1 (XBP1)-EGFP, a marker of endoplasmic reticulum (ER) stress. (A) The XBP1-EGFP marker is activated by $\alpha$-1-antitrypsin (A1AT) null Hong Kong (NHK) allele expression or by treatment with 1 mM dithiothreitol (DTT) treatment, but not by the expression of the ATZ allele. XBP1 splicing was evaluated by western blot analysis using anti-GFP antibodies (upper panel). The levels of ATZ and NHK expression were determined using anti-A1AT antibodies (lower panel). (B and C) Solubility of ATZ and NHK. Drosophila S2 cells transfected with ATZ or NHK were treated with cycloheximide $(3 \mu \mathrm{g} / \mathrm{ml})$ for $0-8 \mathrm{~h}$. The cells were separated into a supernatant and pellet fraction. (B) While western blot analysis with anti-A1AT antibody detected ATZ in both fractions, (C) almost all NHK was observed in the soluble (supernatant) fraction. Anti-profilin was used as a loading control.

Drosophila EDEMs under ER stress conditions, we treated the Drosophila S2 cells with dsRNAs that target either EGFP (as a control), EDEM1 or EDEM2, or both EDEM1 and EDEM2 and subsequently exposed them to $\mathrm{Tg}$. The level of Hsc3 increased after $4 \mathrm{~h}$, and this increase was even more pronounced when both EDEM1 and EDEM2 were knocked down (Fig. 2D). These results suggest that Drosophila EDEMs play protective roles against ER stress.

The ER stress reporter is activated by NHK, but not by the $A T Z$ variant. Excessive protein misfolding in the ER triggers the activation of signaling pathways referred to as the unfolded protein response (UPR). One such pathway involves the mRNA splicing of X-box binding protein 1 (XBP1), which causes a shift in the reading frame of the downstream sequences and the generation of a distinct protein isoform $(17,23)$. We have previously exploited this property to develop a UPR sensor, XBP1-EGFP, in which EGFP is expressed in frame when UPR is activated (17). We thus employed this UPR sensor to assess whether the mutant variants of A1AT cause ER stress in Drosophila. As we have reported previously (14), this UPR marker was activated by NHK expression (Fig. 3A, lane 4) to a similar extent as that induced by DTT treatment (Fig. 3A, lane 6). Intriguingly, ATZ expression in the Drosophila S2 cells did not trigger XBP1 mRNA splicing (Fig. 3A, lane 5). Previous studies on mammalian cells have also reported that, for some reason, ATZ does not activate UPR; instead, ATZ expression activates $\mathrm{NF}-\kappa \mathrm{B}$ signaling via ER overload response (EOR) and ERK signaling (24-26). To determine whether this difference is derived from the solubility of misfolded A1AT, we simply fractionated the cell extracts as supernatants and pellets. We found that the ratio of ATZ protein in the soluble versus the insoluble fraction was roughly 1:1 (Fig. 3B). On the other hand, the majority of NHK protein was found in the soluble fraction (Fig. 3C). These observations support the hypothesis that the two disease alleles of A1AT have distinct biochemical properties.

The degradation of mutant variants of AlAT is regulated by Drosophila EDEMs. We have previously demonstrated that Drosophila EDEM1 and EDEM2 are homologs of mammalian EDEM2 and EDEM3, respectively. Moreover, we demonstrated that the overexpression of Drosophila EDEMs helps to lower the levels of the A1AT NHK variant (14). In this study, to investigate whether Drosophila EDEMs also show distinct specificity toward two misfolded A1AT variants, we examined the effects of EDEM1 and EDEM2 on the degradation of the misfolded ATZ and NHK variants. The level of ATZ increased by approximately 2-fold after the knockdown of EDEM1 and EDEM2 by dsRNA in Drosophila S2 cells (Fig. 4A and B). Similarly, the knockdown of Drosophila EDEM1 and EDEM2 increased the levels of another misfolded A1AT variant, NHK (Fig. 4D and E). Of note, a slightly higher molecular weight band of ATZ was detected after the knockdown of either Drosophila EDEM1 or EDEM2. As the deglycosylation of ERAD substrates occur after being dislocated back into the cytoplasm (27), the slower migrating A1AT band suggests defective ERAD and the accumulation of glycosylated ATZ species that accumulate in the ER.

We also overexpressed EDEMs and found that mutant A1AT degradation was accelerated by the overexpression of EDEMs. Intriguingly, the two EDEMs from Drosophila had additive effects on the degradation of both the NHK (Fig. 5A) and ATZ variants of A1AT (Fig. 5A). Subsequently, we wished to determine whether Drosophila EDEM1 or EDEM2 affects the solubility of ATZ or NHK. We did not observe any signifi- 
A

dsRNA EGFP EDEM1EDEM2

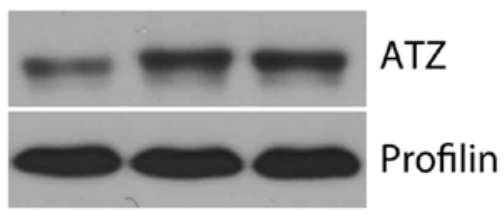

B

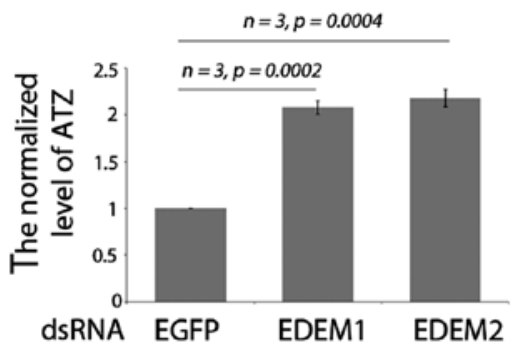

C

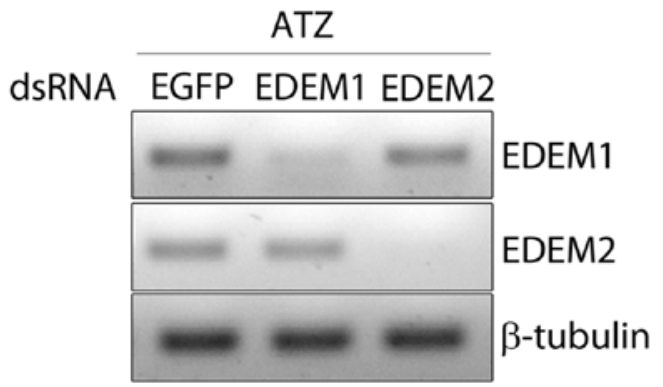

dsRNA EGFP EDEM1EDEM2

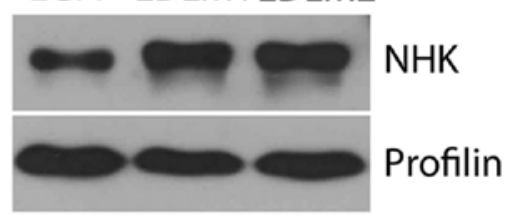

E

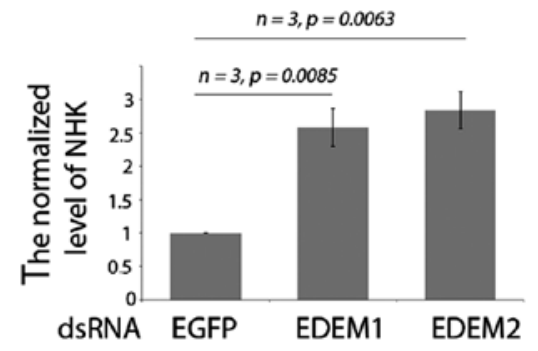

$\mathrm{F}$

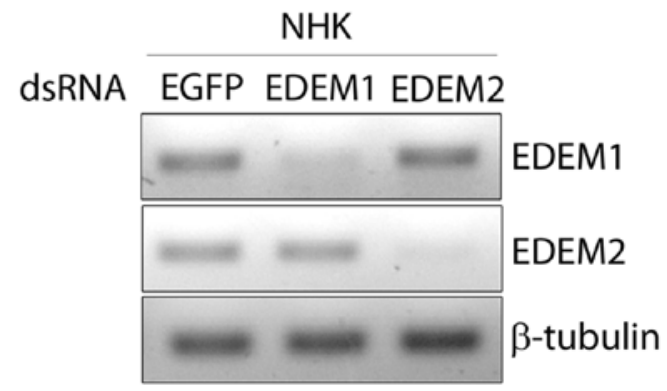

Figure 4. The knockdown of Drosophila endoplasmic reticulum (ER) degradation-enhancing $\alpha$-mannosidase-like proteins (EDEMs) increases the level of mutant $\alpha$-1-antitrypsin (A1AT) proteins. (A and D) The levels of the Z variant of $\alpha$-1-antitrypsin Z (ATZ) and null Hong Kong (NHK) increased following treatment with dsRNAs that target Drosophila EDEM1 or EDEM2. (B and E) The fold change of misfolded A1AT expression level (n=3) was quantified using ImageJ software. The blot of profilin was used to normalize the signal of A1AT. Error bars represent the means \pm SEM. (C and F) EDEM1 and EDEM2 mRNA levels were assessed by RT-PCR.

cant change in the solubility of ATZ or NHK by knocking down EDEM1 or EDEM2 (Fig. 5B and C). These results indicate that Drosophila EDEMs regulate the degradation of misfolded A1AT variants without affecting the solubility of misfolded A1AT.

Drosophila EDEMs increase the level of ubiquitinated misfolded AlAT variants. Previous studies have suggested that ATZ can be degraded by either the ubiquitin-proteasomal pathway, or through autophagy $(13,28,29)$. In order to further confirm that the Drosophila EDEMs act by stimulating the ubiquitin proteasomal degradation of misfolded A1AT variants, we co-expressed Drosophila EDEMs and ATZ with HA-tagged ubiquitin. The co-expression of EDEM1 or EDEM2 with ATZ increased the level of ubiquitinated ATZ (Fig. 5D). The levels of ubiquitinated NHK also increased, albeit to a different extent than that observed for ATZ (Fig. 5E). These results again suggest that Drosophila EDEM1 and EDEM2 target misfolded A1AT variants for proteasomal degradation.

\section{Discussion}

In the present study, we report the use of a Drosophila model for the study of the mechanisms of misfolded A1AT degradation that underly A1AT deficiency (30-32). Specifically, we focused on EDEMs, which are ER resident proteins with mannosidaselike domains. Similar to the mammalian EDEMs, we find that the Drosophila EDEM2 mRNA level increases in response to ER stress. We did not observe a similar induction with the EDEM1 mRNA level. The examination of the temporal and tissue-specific expression of Drosophila EDEM2 indicated that the tissues with the highest levels of Drosophila EDEM2 transcripts include the larval salivary gland, the adult intestine and the fat body, all of which have a high protein secretion load (33). Of note, these three organs are all characterized by high levels of IRE1/XBP1 activity $(17,34)$. As mammalian EDEMs are regulated by IRE1/XBP1 signaling, it is likely that the Drosophila IRE1/XBP1 pathway contributes to the induction of EDEM2 during specific developmental stages, as well as upon ER stress.

EDEMs are involved in one of the early steps of ERAD substrate recognition. The tight regulation of ERAD is important as the inefficient detection of misfolded or unfolded proteins causes their accumulation in the ER, and leads to ER stress. On the other hand, overactive ERAD can degrade ER resident proteins or folding intermediates. Although the expression of the majority of ERAD components is upregulated by ER stress, we observed a significantly high level of Drosophila EDEM1 transcripts even under conditions of no 
A

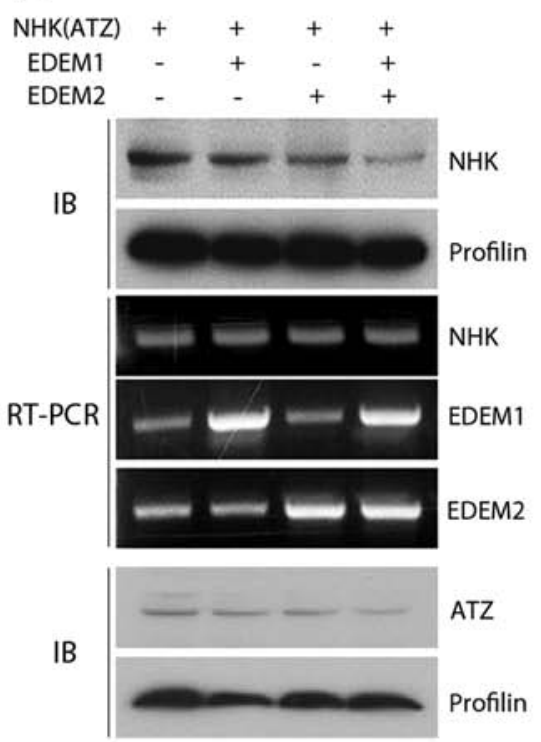

D

$\begin{array}{cccc}\text { ATZ } & + & + & + \\ \text { EGFP } & + & - & - \\ \text { EDEM1 } & - & + & - \\ \text { EDEM2 } & - & - & + \\ \text { HA-ubi } & + & + & +\end{array}$

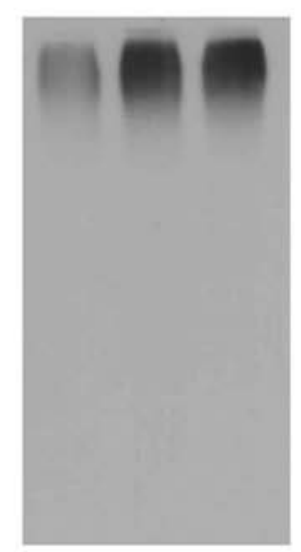

$\mathrm{E}$

$\begin{array}{lccc}\text { NHK } & + & + & + \\ \text { EGFP } & + & - & - \\ \text { EDEM1 } & - & + & - \\ \text { EDEM2 } & - & - & + \\ \text { HA-ubi } & + & + & +\end{array}$

IP : anti-A1AT

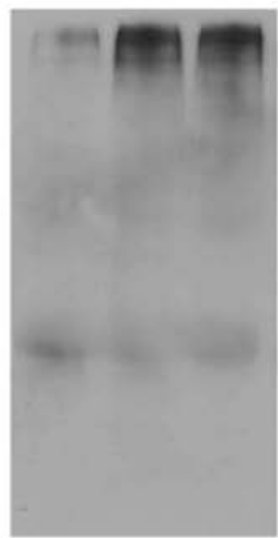

IP : anti-A1AT

IB : anti-HA

B

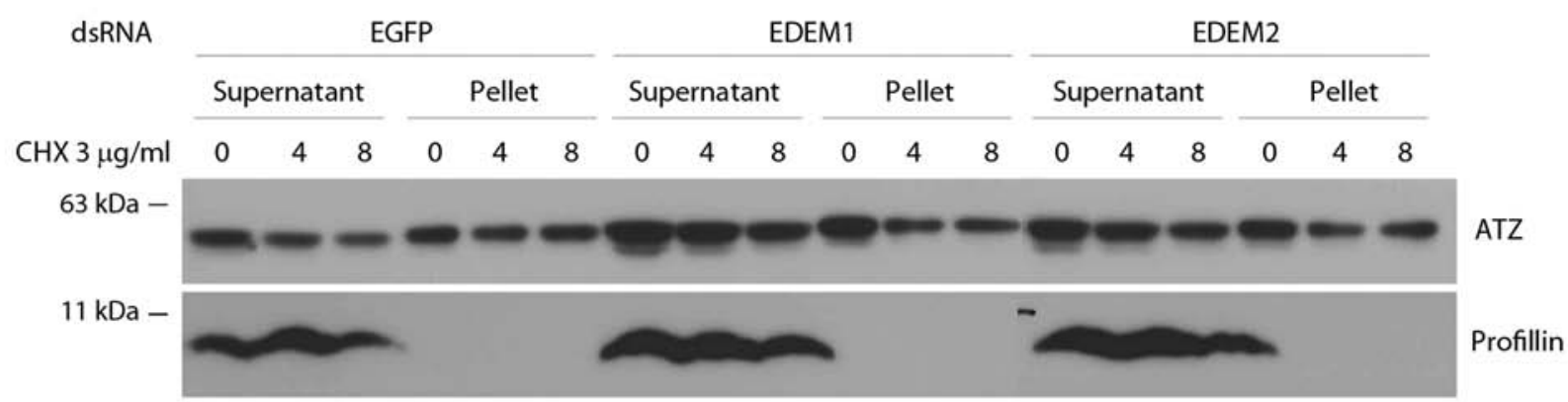

C

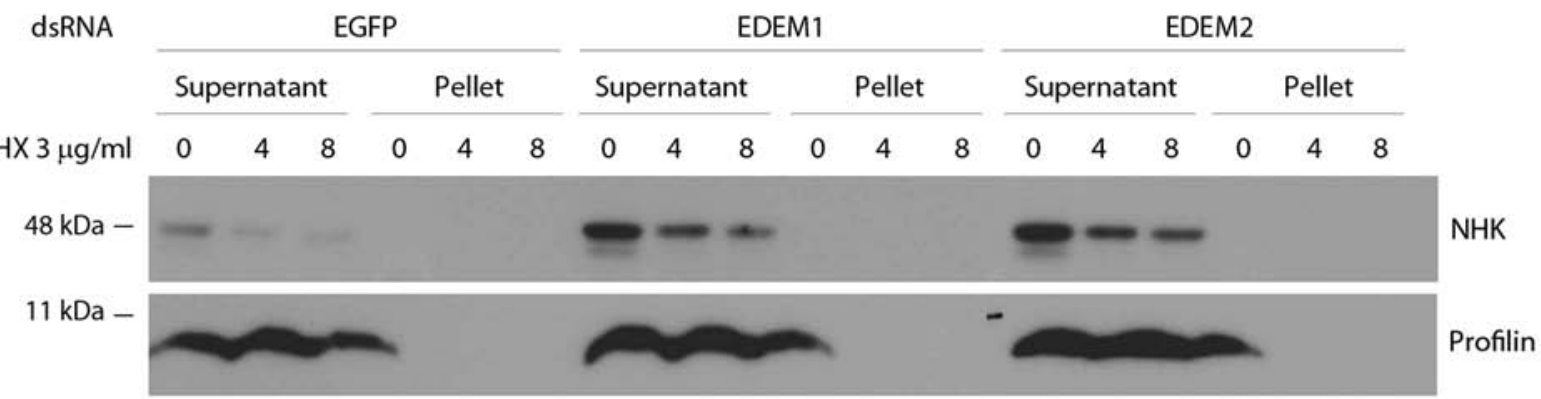

Figure 5. Drosophila endoplasmic reticulum (ER) degradation-enhancing $\alpha$-mannosidase-like proteins (EDEMs) increase the degradation of misfolded $\alpha$-1-antitrypsin (A1AT). (A) The A1AT variant was coexpressed with EDEM1 or EDEM2 in Drosophila S2 cells. A1AT transcript levels were assessed by RT-PCR, and protein levels were assessed by western blot analyses. (B and C) The solubility of $\alpha$-1-antitrypsin Z (ATZ) or null Hong Kong (NHK) after the knockdown of Drosophila EDEM1 or EDEM2. Drosophila EDEMs did not have an obvious effect on the solubility of misfolded A1AT. The level of (B) ATZ or (C) NHK was analyzed in cells pre-treated with dsRNAs against EGFP (lanes 1-6), EDEM1 dsRNA (lanes 7-12), or EDEM2 (lanes 13-18). The lower gel shows anti-profilin bands as loading controls. (D and E) The ubiquitination of ATZ and NHK in transfected cells. Drosophila S2 cells were transfected with plasmids encoding HA-tagged ubiquitin and either ATZ or NHK together with Drosophila EDEM1 or EDEM2. (D) ATZ or (E) NHK was immunoprecipitated using anti-A1AT antibody, and the degree of ubiquitination of ATZ or NHK was analyzed by western blot analysis using the anti-HA antibody. IP, immunoprecipitation.

stress (Fig. 2D). The mechanisms through which EDEM1 distinguishes terminally misfolded proteins versus folding intermediates remains to be explored.

In conclusion, the results from the present study indicate that the Drosophila EDEMs, EDEM1 and EDEM2, are resident in the ER, and that the expression of Drosophila EDEM2 is upregulated by ER stress. Both EDEM1 and EDEM2 in Drosophila promote the degradation of misfolded A1AT variants by increasing the ubiquitination of its substrates. Given the striking similarity between Drosophila and humans in terms of this process, the present study provides a novel approach for the study of A1AT deficiency. 


\section{Acknowledgements}

We would like to thank the Kang laboratory for their helpful comments on the manuscript. This study was supported by grants from the Korean Health Technology R\&D Project, Ministry of Health and Welfare, Republic of Korea (HI13C1821), the National Research Foundation of Korea (NRF-2013R1A1A1009437), the Korean Government (MSIP) (MRC grant 2008-0062286) to M.-J.K. and the National Institutes of Health grant R01 EY020866 to H.D.R.

\section{References}

1. Vembar SS and Brodsky JL: One step at a time: Endoplasmic reticulum-associated degradation. Nat Rev Mol Cell Biol 9: 944-957, 2008.

2. Molinari M, Calanca V, Galli C, Lucca P and Paganetti P: Role of EDEM in the release of misfolded glycoproteins from the calnexin cycle. Science 299: 1397-1400, 2003.

3. Oda Y,Hosokawa N, Wada I and Nagata K: EDEM as an acceptor of terminally misfolded glycoproteins released from calnexin. Science 299: 1394-1397, 2003.

4. Clerc S, Hirsch C, Oggier DM, Deprez P, Jakob C, Sommer T and Aebi M: Htm1 protein generates the N-glycan signal for glycoprotein degradation in the endoplasmic reticulum. J Cell Biol 184: 159-172, 2009.

5. Hosokawa N, Tremblay LO, Sleno B, Kamiya Y, Wada I, Nagata K, Kato K and Herscovics A: EDEM1 accelerates the trimming of alpha1,2-linked mannose on the $\mathrm{C}$ branch of N-glycans. Glycobiology 20: 567-575, 2010.

6. Hebert DN and Molinari M: Flagging and docking: Dual roles for $\mathrm{N}$-glycans in protein quality control and cellular proteostasis. Trends Biochem Sci 37: 404-410, 2012.

7. Lomas DA, Evans DL, Finch JT and Carrell RW: The mechanism of $\mathrm{Z}$ alpha 1-antitrypsin accumulation in the liver. Nature 357: 605-607, 1992.

8. Termine DJ, Moremen KW and Sifers RN: The mammalian UPR boosts glycoprotein ERAD by suppressing the proteolytic downregulation of ER mannosidase I. J Cell Sci 122: 976-984, 2009.

9. Wu Y, Swulius MT, Moremen KW and Sifers RN: Elucidation of the molecular logic by which misfolded alpha 1-antitrypsin is preferentially selected for degradation. Proc Natl Acad Sci USA 100: 8229-8234, 2003.

10. Mast SW, Diekman K, Karaveg K, Davis A, Sifers RN and Moremen KW: Human EDEM2, a novel homolog of family 47 glycosidases, is involved in ER-associated degradation of glycoproteins. Glycobiology 15: 421-436, 2005.

11. Teckman JH and Perlmutter DH: Retention of mutant alpha(1)antitrypsin $\mathrm{Z}$ in endoplasmic reticulum is associated with an autophagic response. Am J Physiol Gastrointest Liver Physiol 279: G961-G974, 2000.

12. Kamimoto T, Shoji S, Hidvegi T, Mizushima N, Umebayashi K, Perlmutter DH and Yoshimori T: Intracellular inclusions containing mutant alpha1-antitrypsin $\mathrm{Z}$ are propagated in the absence of autophagic activity. J Biol Chem 281: 4467-4476, 2006

13. Kroeger H, Miranda E, MacLeod I, Pérez J, Crowther DC Marciniak SJ and Lomas DA: Endoplasmic reticulum-associated degradation (ERAD) and autophagy cooperate to degrade polymerogenic mutant serpins. J Biol Chem 284: 22793-22802, 2009.

14. Kang MJ and Ryoo HD: Suppression of retinal degeneration in Drosophila by stimulation of ER-associated degradation. Proc Natl Acad Sci USA 106: 17043-17048, 2009.

15. Hosokawa N, Tremblay LO, You Z, Herscovics A, Wada I and Nagata K: Enhancement of endoplasmic reticulum (ER) degradation of misfolded Null Hong Kong alpha1-antitrypsin by human ER mannosidase I. J Biol Chem 278: 26287-26294, 2003

16. Brand AH and Perrimon N: Targeted gene expression as a means of altering cell fates and generating dominant phenotypes. Development 118: 401-415, 1993.
17. Ryoo HD, Domingos PM, Kang MJ and Steller H: Unfolded protein response in a Drosophila model for retinal degeneration. EMBO J 26: 242-252, 2007.

18. Wu Y, Whitman I, Molmenti E, Moore K, Hippenmeyer P and Perlmutter DH: A lag in intracellular degradation of mutant alpha 1-antitrypsin correlates with the liver disease phenotype in homozygous PiZZ alpha 1-antitrypsin deficiency. Proc Natl Acad Sci USA 91: 9014-9018, 1994.

19. Armknecht S, Boutros M, Kiger A, Nybakken K, Mathey-Prevot B and Perrimon N: High-throughput RNA interference screens in Drosophila tissue culture cells. Methods Enzymol 392: 55-73, 2005.

20. Shen Y, Ballar P and Fang S: Ubiquitin ligase gp78 increases solubility and facilitates degradation of the $\mathrm{Z}$ variant of alpha-1-antitrypsin. Biochem Biophys Res Commun 349: 1285-1293, 2006.

21. Olivari S, Galli C, Alanen H, Ruddock L and Molinari M: A novel stress-induced EDEM variant regulating endoplasmic reticulum-associated glycoprotein degradation. J Biol Chem 280: 2424-2428, 2005.

22. Hosokawa N, Wada I, Hasegawa K, Yorihuzi T, Tremblay LO, Herscovics A and Nagata K: A novel ER alpha-mannosidase-like protein accelerates ER-associated degradation. EMBO Rep 2: 415-422, 2001.

23. Coelho DS, Cairrão F, Zeng X, Pires E, Coelho AV, Ron D, Ryoo HD and Domingos PM: Xbpl-independent Ire1 signaling is required for photoreceptor differentiation and rhabdomere morphogenesis in Drosophila. Cell Rep 5: 791-801, 2013.

24. Hidvegi T, Schmidt BZ, Hale P and Perlmutter DH: Accumulation of mutant alpha1-antitrypsin $\mathrm{Z}$ in the endoplasmic reticulum activates caspases- 4 and -12, NFkappaB, and BAP31 but not the unfolded protein response. J Biol Chem 280: 39002-39015, 2005.

25. Lawless MW, Greene CM, Mulgrew A, Taggart CC, O'Neill SJ and McElvaney NG: Activation of endoplasmic reticulum-specific stress responses associated with the conformational disease $\mathrm{Z}$ alpha 1-antitrypsin deficiency. J Immunol 172: 5722-5726, 2004.

26. van 't Wout EF, Dickens JA, van Schadewijk A, et al: Increased ERK signalling promotes inflammatory signalling in primary airway epithelial cells expressing $\mathrm{Z} \alpha 1$-antitrypsin. Hum Mol Genet 23: 929-941, 2014.

27. Hirsch C, Blom D and Ploegh HL: A role for N-glycanase in the cytosolic turnover of glycoproteins. EMBO J 22: 1036-1046, 2003.

28. Liu Y, Choudhury P, Cabral CM and Sifers RN: Oligosaccharide modification in the early secretory pathway directs the selection of a misfolded glycoprotein for degradation by the proteasome. J Biol Chem 274: 5861-5867, 1999.

29. Teckman JH, Gilmore R and Perlmutter DH: Role of ubiquitin in proteasomal degradation of mutant alpha(1)-antitrypsin $\mathrm{Z}$ in the endoplasmic reticulum. Am J Physiol Gastrointest Liver Physiol 278: G39-G48, 2000.

30. Perlmutter DH: Liver injury in alpha1-antitrypsin deficiency: An aggregated protein induces mitochondrial injury. J Clin Invest 110: 1579-1583, 2002.

31. Lin L, Schmidt B, Teckman J and Perlmutter DH: A naturally occurring nonpolymerogenic mutant of alpha 1-antitrypsin characterized by prolonged retention in the endoplasmic reticulum. $\mathrm{J}$ Biol Chem 276: 33893-33898, 2001.

32. Parfrey H, Mahadeva R and Lomas DA: Alpha(1)-antitrypsin deficiency, liver disease and emphysema. Int J Biochem Cell Biol 35: 1009-1014, 2003.

33. Gelbart WM and Emmert DB: FlyBase high throughput expression pattern data. flybase.org/reports/FBrf0221009. html.2013.

34. Sone M, Zeng X, Larese J and Ryoo HD: A modified UPR stress sensing system reveals a novel tissue distribution of IRE1/XBP1 activity during normal Drosophila development. Cell Stress Chaperones 18: 307-319, 2013. 